\title{
Intra-arterial administration of adriamycin-loaded albumin microspheres for locally advanced breast
} cancer

\author{
JC Doughty, JH Anderson, N Willmott, CS McArdle
}

\begin{abstract}
Summary
Regional chemotherapy is an attractive but underevaluated method of treating locally advanced breast cancer. We have combined two novel methods of targetting by delivering a single pulse of adriamycin-loaded albumin microspheres down a radiologically placed internal mammary artery catheter. A complete response was observed and prolonged local control achieved.
\end{abstract}

Keywords: breast carcinoma, chemotherapy

\section{Introduction}

Despite increasing public awareness $15 \%$ of patients with breast cancer still present with locally advanced disease. The five-year survival for these patients treated by local therapy alone is only $20 \%$, and in an attempt to address this problem systemic therapy is now routinely administered to these patients. Although there has been a wealth of clinical trials showing encouraging response rates and five-year survivals, most of these trials have been nonrandomised and contain small numbers of patients.

EORTC, however, randomised 363 patients to receive either radiotherapy, radiotherapy plus hormonal therapy, radiotherapy plus chemotherapy or radiotherapy plus a combination of hormonal and chemotherapy. The results of this study showed that the time to first progression was significantly delayed by the addition of systemic therapy. The effect was due almost entirely to the time to locoregional progression rather than time to distant metastases. There was no significant difference in overall survival between the groups. ${ }^{1}$

Since most drugs are dose-dependent, regional chemotherapy which aims to deliver high doses of drug directly to the tumour by first-order targeting may be a more effective treatment. In the breast it is conventionally delivered through a catheter surgically implanted directly into either the subclavian artery or into a branch of the subclavian artery, usually the thyrocervical trunk. ${ }^{2}$ More selective perfusion, however, can be obtained by angiographic placement of catheters directly into the internal mammary artery. ${ }^{3}$

Targeting can be further enhanced by the use of cytotoxin-loaded microspheres. When administered intra-arterially these microspheres are carried by the blood flow to the capillary bed where they embolise and release their therapeutic pay-load into the target organ. ${ }^{4}$ Although no randomised clinical trials have been undertaken, animal studies have shown that adriamycin-loaded albumin microspheres can suppress tumour growth to a greater degree than free drug in solution. ${ }^{5}$

We have combined both of these methods of targeting by delivering a single pulse of adriamycin-loaded microspheres down a radiologically placed internal mammary artery catheter in a patient with locally advanced breast cancer. A complete response was observed and prolonged local control was achieved until the patient's death from disseminated disease.

\section{Case report}

A 42-year-old woman presented with a 14-cm stage III left breast cancer which was fixed to the skin and underlying muscle. Diagnosis was confirmed by 'trucut' biopsy. Staging at that time showed no evidence of axillary lymphadenopathy or systemic disease. Using a modified Seldinger technique, a catheter was inserted radiologically into the left internal mammary artery and $9 \mathrm{mg}$ of doxorubicin in $90 \mathrm{mg}$ of freeze-dried albumin microspheres with a $10 \%$ drug pay-load were administered over a 10-minute period. This dose was less than the normal systemic dose as we were delivering a greater proportion of drug directly to the breast and did not want to experience local toxicity. The distribution of the microspheres is seen in figure 1 . As well as being distributed to the breast, staining was also visible in the upper quadrant of the abdomen in the distribution of the superior epigastric artery. No systemic toxicity was observed.

After two months, clinical assessment revealed no residual tumour palpable in the breast. However, necrosis of the subcutaneous fat over the left upper quadrant of the abdomen had occurred, leading to three small ulcers and a sinus, which delayed the administration of 'adjuvant' systemic chemotherapy (CMF), and planned radiotherapy. At six months bone metastases developed and at 12 months a metastasis of the opposite breast. Twenty three months after presentation the patient 


\begin{tabular}{|l|}
\hline Treatment options for locally \\
advanced breast carcinoma \\
\hline - surgery \\
- radiotherapy \\
- systemic chemotherapy \\
- regional chemotherapy \\
\hline
\end{tabular}

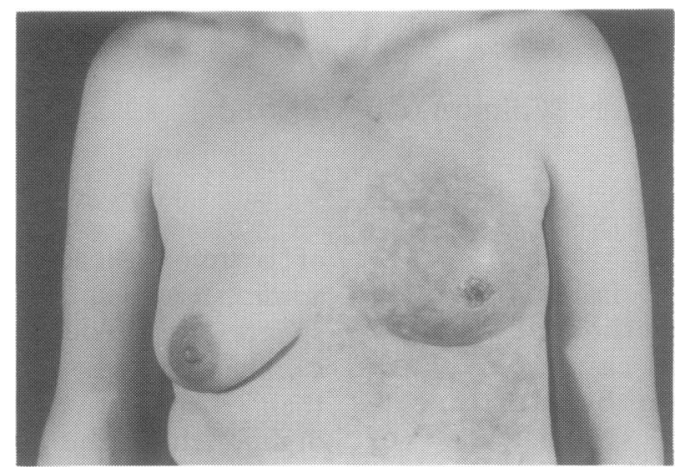

Figure 1 Skin staining indicating area of distribution of the microspheres with ulceration of the anterior abdominal wall.

developed cerebral metastases and died. Throughout this time good control was obtained in the left breast and no residual tumour could be palpated.

\section{Discussion}

Management of locally advanced breast cancer remains a difficult clinical problem, and the optimal treatment has yet to be determined. Systemic chemotherapy in combination with radiotherapy and surgery has produced impressive response rates but some series report a local recurrence rate of up to $38 \%$ and toxicity is high with these regimes. ${ }^{6}$ The five-year survival of patients presenting with locally advanced disease treated by surgery alone is only $25 \%$, and this reflects the high proportion of patients who have micrometastatic disease at presentation. Nevertheless an important aim of treatment should be to gain local control.

Regional chemotherapy is an attractive concept which aims to increase the concentration of drug entering the tumour-bearing organ by directly infusing drug via its arterial supply. The amount of drug entering the systemic circulation will be dependent upon the degree of uptake and metabolism of the drug by the target organ. Conventionally regional chemotherapy to the breast is delivered by surgical implantation of a catheter directly into the subclavian artery or into a branch of the

1 Rubens RD, Bartelink H, Engelsman E, et al. Locally advanced breast cancer: the contribution of cytotoxic and endocrine treatment to radiotherapy. An EORTC Breast Cancer Co-operative Group Trial (10792). Eur $\mathcal{f}$ Cancer Clin Oncol 1989; 25: 667-8.

2 Aigner KR, Walther $H$, Muller $H$, Jansa J, Thiem $N$. Intra-arterial infusion chemotherapy for recurrent breast cancer via an implantable system. Reg Cancer Treatment 1988; 1: 102-7. subclavian artery, usually the thyrocervical trunk. ${ }^{2}$ A tourniquet is applied to prevent the chemotherapy perfusing the arm, limiting the infusion to 15 minutes. However, using this method unwanted perfusion of the neck back and upper trunk occurs. A more selective approach is to place catheters angiographically into the internal mammary artery as in this patient.

The anthracyclines have been shown to be the most effective single agent in the treatment of breast cancer, ${ }^{7}$ and in the absence of the development of new drugs, methods have been devised to increase delivery of these drugs to the tumour. Evidence from animal models suggests that drug-loaded microspheres are more potent than the same concentration of drug in solution, ${ }^{5}$ and it is the sustained-release properties of microspheres rather than induction of reductive drug metabolism which is important for anti-tumour activity. ${ }^{8}$

We have used two novel methods of targetting to treat a patient with locally advanced breast cancer. Although an impressive clinical response was obtained, it was complicated by the development of ulceration of the anterior abdominal wall. This occurs because the internal mammary artery terminates by dividing into the superior epigastric and musculophrenic arteries and chemotherapy delivered this way perfuses the areas supplied by these vessels. The breast is a highly vascular organ and microspheres directed towards the breast will block the first capillary bed they reach and will remain in breast tissue. Microspheres flowing down the superior epigastric artery will reach their first capillary bed in the skin and, if adjacent areas of skin are embolised, confluent ischaemic ulcers may occur which take many months to heal and may delay further regional treatment. Phrenic nerve paralysis secondary to chemotherapy flowing down the musculophrenic artery following internal mammary artery perfusion has also been reported. ${ }^{9} \mathrm{We}$ have since devised a technique of angiographically placing an occlusion coil in the internal mammary artery distal to the last branch to the breast. This eliminates unwanted perfusion of the diaphragm and upper abdominal wall. ${ }^{10}$

This case illustrates the value of combining two novel techniques to improve targetting to tumour. A good quality prolonged response was achieved with a single pulse of therapy without systemic toxicity. The only disadvantage of this approach, namely unwanted perfusion of the diaphragm and anterior abdominal wall, is now preventable.

Mr JH Anderson was sponsored by the CRC.

3 Murata M, Takagi S, Seki M, Tezuka T, Shinohara K Miura T. A case of complete response of locally advanced breast cancer to arterial infusion chemotherapy cannulation of the right femoral artery and combined use of G-CSF Gan-To-Kagaku-Ryoho 1992; 19: 1625-7.

4 McArdle CS, Hansell D, Kerr DJ, McKillop J, Willmott N. Cytotoxic-loaded albumin microspheres: a novel approach to regional chemotherapy. $B r \mathcal{F}$ Surg 1988; 75: 132-4. 
5 Goldberg JA, Willmott N, Kerr DJ, Sutherland C, McArdle CA. An in vivo assessment of adriamycin-loaded albumin microspheres. Br F Cancer 1992; 65: 393-5.

6 Booser DJ, Hortobagyi GN. Treatment of locally advanced breast cancer. Semin Oncol 1992; 19; 278-85.

7 Willmott N, Cummings J, Marley E, Smyth JF. Relationship between reductive drug metabolism in tumour tissue of anthracyclines in microspherical form and anti-tumour anthracyclines in microspherical form and
activity. Biochem Pharmacol 1990; 39: 1055-?

8 Harris JR, Canellos GP, Hellman S, Fisher B. In: De Vita

8 Harris JR, Canellos GP, Hellman S, Fisher B. In: De Vita phia: Lippincott, 1985; p 1156.
9 Twelves CJ, Chaudray MA, Reidy J, et al. Toxicity of intra-arterial doxorubicin in locally advanced breast cancer. Cancer Chemother Pharmacol 1990; 25: 459-62.

10 McCarter DHA, Doughty JC, McArdle CS, Cooke TC Reid AW. Angiographic embolisation of the distal internal mammary artery as an adjunct to regional chemotherapy in cular Intervent Radiol, in press.

\title{
Anomalous splenic artery aneurysm: demonstration on CT scanning and angiography
}

\author{
Paul S Sidhu, Kok-Tee Khaw, Anna-Maria Belli
}

\begin{abstract}
Summary
An asymptomatic 35-year-old female was found to have a congenital aneursym of an anomalous splenic artery arising from the superior mesenteric artery on routine investigation for infertility. Demonstration on CT and angiography is presented and the pathogenesis, natural history, management, and implications during pregnancy are discussed.
\end{abstract}

Keywords: splenic artery, aneurism, CT scanning, angiography

\section{Introduction}

Aneurysms arising from the splenic artery are uncommon and are usually removed surgically or embolised to prevent the occurrence of rupture and sudden death. The splenic artery has its usual origin from the coeliac axis but rarely arises from other sites including the superior mesenteric artery (SMA). We describe a case of an anomalous splenic artery derived from the SMA with a congenital saccular aneurysm arising from it.

\section{Case report}

Department of Diagnostic Radiology, Hammersmith Hospital, DuCane Road, London W12 OHS, UK

PS Sidhu A-M Belli

\section{Radiology} Department, Ealing Hospital, London, UK K-T Khaw

\section{Correspondence to} Dr PS Sidhu, 1 A Coleraine Road, Blackheath, London SE3 7PF, UK
An asymptomatic 35-year-old Asian female patient with mild idiopathic thrombocytopaenia purpura (ITP) attended the haematology clinic on a six-monthly basis. The patient was anaemic $(10.3 \mathrm{~g} / \mathrm{dl})$ with a mildly low platelet count $\left(114 \times 10^{9} / 1\right)$ and no other overt manifestations or symptoms attributable to the diagnosis of ITP. Clinical examination of the patient was entirely normal. The patient did, however, complain of infertility. As part of the routine investigations for infertility the patient had an abdominal/pelvic ultrasound. On the B-mode ultrasound examination, a 2-cm rounded echo-poor area thought to be contiguous with the SMA was demonstrated. A subsequent CT scan (Toshiba Xpeed) demonstrated a $2-\mathrm{cm}$ rounded area arising from the
$A$

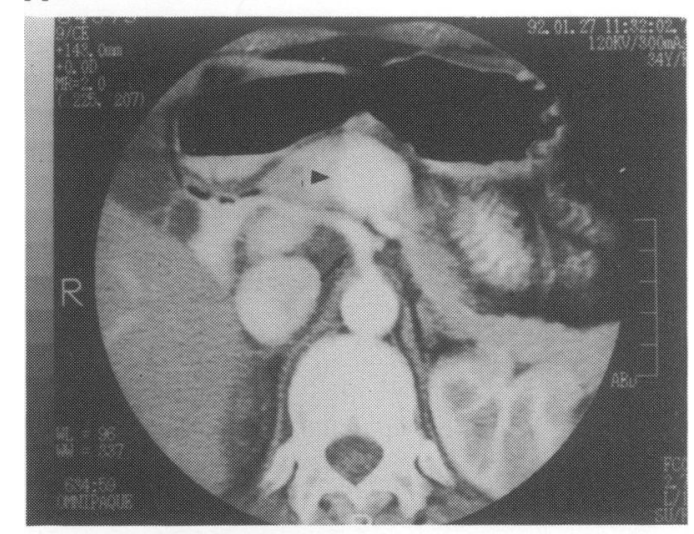

B

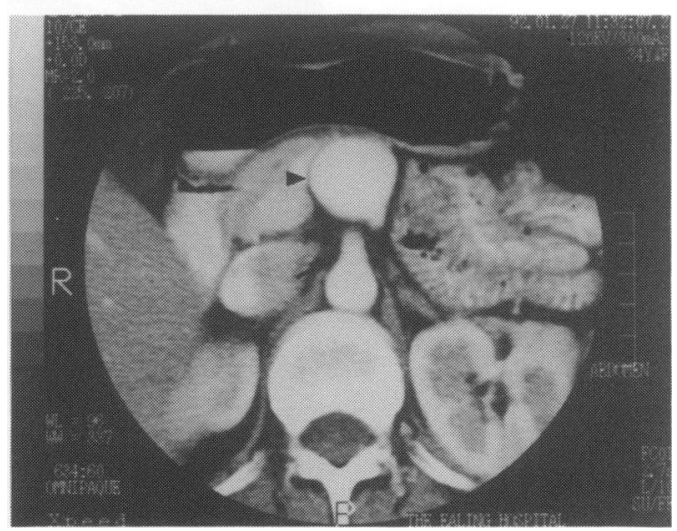

Figure 1 (A) Magnified view of a transaxial section at the level of the coeliac axis (arrow) demonstrating the common hepatic artery (arrow) and a $2-\mathrm{cm}$ vascular aneurysm lying anterior (arrowhead). No splenic artery arises from the coeliac axis. (B) $10 \mathrm{~mm}$ caudal to the level in (A); the origin of the SMA (arrow) and the vascular aneurysm (arrowhead) are seen

splenic artery with uniform enhancement after the administration of contrast (Omnipaque 300) compatible with a vascular aneurysm (figure 1A and B). A Digital Subtraction Angiogram (DSA) performed via selective catheterisation of the SMA, demonstrated an 